\title{
Near-real-time tracking of gaps in prevention of mother-to-child transmission of HIV in three districts of KwaZulu-Natal Province, South Africa
}

\author{
F Moyo, ${ }^{1,2,6}$ MSc; A Haeri Mazanderani, ${ }^{2,3}$ MB ChB; S Bhardwaj, ${ }^{4}$ MB BS, MD, MPH; O B Mhlongo, ${ }^{5}$ B Cur; T Kufa, ${ }^{2,6}$ MB ChB, MPH, PhD; \\ K Ng'oma, ${ }^{4}$ MB BS, MSc; B A Smith, ${ }^{4}$ B Tech; G G Sherman, ${ }^{1,2,7}$ MB BCh, MMed, PhD \\ ${ }^{1}$ Paediatric HIV Diagnostics, Wits Health Consortium, Johannesburg, South Africa \\ ${ }^{2}$ Centre for HIV and STIs, National Institute for Communicable Diseases, National Health Laboratory Service, Johannesburg, South Africa \\ ${ }^{3}$ Department of Medical Virology, Faculty of Health Sciences, University of Pretoria, South Africa \\ ${ }^{4}$ United Nations Children's Fund (UNICEF), Pretoria, South Africa \\ ${ }^{5}$ National Department of Health, KwaZulu-Natal Province, South Africa \\ ${ }^{6}$ School of Public Health, Faculty of Health Sciences, University of the Witwatersrand, Johannesburg, South Africa \\ ${ }^{7}$ Department of Paediatrics and Child Health, Faculty of Health Sciences, University of the Witwatersrand, Johannesburg, South Africa
}

Corresponding author: F Moyo (faithmo@nicd.ac.za)

\begin{abstract}
Background. Identifying and addressing gaps in the prevention of mother-to-child transmission of HIV (PMTCT) is required if South Africa (SA) is to achieve targets for eliminating MTCT (eMTCT). Potential PMTCT gaps that increase MTCT risk include late maternal HIV diagnosis, lack of or delayed antiretroviral therapy (ART) during pregnancy and breastfeeding, and lack of effective prophylaxis for HIV-exposed infants.

Objectives. To investigate, in near real time, PMTCT gaps among HIV-infected infants in three districts of KwaZulu-Natal Province, SA.

Methods. Between May and September 2016, PMTCT co-ordinators from eThekwini, uMgungundlovu and uMkhanyakude districts received daily email notification of all HIV polymerase chain reaction (PCR)-positive results. Co-ordinators reviewed facility records for each infant to identify gaps in PMTCT care, including maternal age, timing of maternal HIV diagnosis, maternal treatment history and maternal viral load (VL) monitoring. Data were submitted via the mobile phone SMS (text message) service using Rapid Pro technology and analysed in Stata 14.

Results. Data on PMTCT gaps were received for 367 (91.8\%) of 400 infants with HIV PCR-positive results, within a median time of 12.5 days (interquartile range (IQR) 6 - 23). The median maternal age was 25 years (IQR 22 - 30), with 48 teenage mothers (15 - 19 years). The sample size was too small to determine whether there were significant differences in PMTCT gaps between the 48 teenage mothers and 293 older (20 - 34 years) mothers. Of the mothers, 220 (60.0\%) were first diagnosed prior to conception or at their first antenatal care (ANC) visit, and $127(34.6 \%)$ at or after delivery; 137 (37.3\%) transmitted HIV to their infants despite receiving $>12$ weeks of ART. VL results were unavailable for $70.0 \%$ of women. Only 41 (17.5\%) of women known to be HIV-positive during ANC had confirmed virological suppression. No statistically significant differences in PMTCT gaps were observed between districts, owing to small sample sizes in uMgungundlovu and uMkhanyakude.

Conclusions. The findings highlight the need to improve services during ANC, in particular prioritising maternal VL monitoring. We intend to use improved technology to streamline data collection and reporting towards eMTCT.
\end{abstract}

S Afr Med J 2018;108(4):319-324. DOI:10.7196/SAMJ.2018.v108i4.12630

The success of South Africa (SA)'s prevention of mother-to-child transmission of HIV (PMTCT) programme is well documented, demonstrating a significant reduction in the early infant transmission rate over the past decade from $>20 \%$ to $<2 \% .^{[1-4]}$ Much of this progress can be attributed to increased access to effective maternal antiretroviral therapy (ART) and improved infant prophylactic regimens, coupled with robust monitoring and evaluation systems. SA's PMTCT guidelines shifted from recommendations in line with World Health Organization (WHO) Option A in 2013, where different ART regimens were provided to mothers based on immunological profile, to Option B, recommending that all HIV-infected pregnant and breastfeeding women be treated with triple ART, and Option B+ in 2015, which advocates lifelong triple ART irrespective of CD4+ cell count or clinical stage. ${ }^{[5-7]}$ Daily infant nevirapine (NVP) prophylaxis for at least 6 weeks' duration has been part of national guidelines since 2010, with infants identified as at high risk recommended either extended daily NVP for 12 weeks or dual prophylaxis with NVP plus zidovudine (AZT) for 6 weeks. ${ }^{[5-7]}$ Table 1 describes the evolution of PMTCT guidelines from 2010 to 2015. ${ }^{[8]}$

Maternal HIV viral load (VL) has been identified as a strong predictor of mother-to-child transmission (MTCT) risk. ${ }^{[9]}$ An infant born to a mother who is not virologically suppressed is at increased risk of infection and requires high-risk prophylaxis. ${ }^{[7]}$ On account of this and the availability of more affordable VL testing, ${ }^{[10]}$ SA reviewed its guidelines for treatment monitoring in 2015, recommending that all women on ART have a VL test done at confirmation of pregnancy, and that women who have been newly initiated on ART have VL testing at 3,6,12,18 and 24 months thereafter throughout pregnancy and breastfeeding. ${ }^{[7]}$ The early infant diagnosis programme has also evolved over the years to enable earlier detection of perinatal 
Table 1. Evolution of the South African PMTCT guidelines for HIV-infected pregnant women and HIV-exposed infants aged $<18$ months, $2010-2015$

\begin{tabular}{|c|c|c|c|}
\hline & 2010, WHO Option A & 2013, WHO Option B & 2015, WHO Option B+ \\
\hline Maternal ART & $\begin{array}{l}\text { 1. If CD4+ }<350 \text { cells/ } \mu \mathrm{L} \text { or WHO } \\
\text { stage III or IV: initiate ART lifelong } \\
\text { 2. If CD }>350 \text { cells/ } \mu \mathrm{L} \text { : } \\
\text { - AZT at } 14 \text { weeks' gestation } \\
\text { - Single-dose NVP + AZT/3TC at } \\
\text { onset of labour } \\
\text { - AZT/3TC daily for } 7 \text { days } \\
\text { postpartum }\end{array}$ & $\begin{array}{l}\text { All pregnant and breastfeeding } \\
\text { HIV-infected women initiated on } \\
\text { ART for duration of pregnancy and } \\
\text { breastfeeding } \\
\text { 1. If CD } 4+>350 \text { cells/ } \mu \mathrm{L} \text { : continue } \\
\text { ART until } 1 \text { week post cessation of } \\
\text { breastfeeding } \\
\text { 2. If CD } 4<350 \text { cells/ } \mu \mathrm{L} \text { : continue ART } \\
\text { lifelong }\end{array}$ & $\begin{array}{l}\text { All pregnant and breastfeeding HIV- } \\
\text { infected women initiated on lifelong } \\
\text { ART }\end{array}$ \\
\hline Infant prophylaxis & $\begin{array}{l}\text { Single-dose NVP daily for } 6 \text { weeks } \\
\text { if mother is on ART or for duration } \\
\text { of breastfeeding }+1 \text { month post } \\
\text { cessation of breastfeeding }\end{array}$ & Single-dose NVP daily for 6 weeks & $\begin{array}{l}\text { 1. Low risk: Daily dose NVP for } \\
6 \text { weeks } \\
\text { 2. High risk: } \\
\text { - NVP for } 12 \text { weeks if mother is } \\
\text { on cART }<4 \text { weeks, or newly } \\
\text { diagnosed at delivery } \\
\text { - NVP + AZT for } 6 \text { weeks if } \\
\text { maternal VL }>1000 \mathrm{cps} / \mathrm{mL} \\
\text { - NVP + AZT if newly diagnosed } \\
\text { while breastfeeding. If HIV PCR is } \\
\text { negative, continue NVP for a total } \\
\text { of } 12 \text { weeks }\end{array}$ \\
\hline
\end{tabular}

PMTCT $=$ prevention of mother-to-child transmission; $\mathrm{WHO}=$ World Health Organization; $\mathrm{ART}=$ antiretroviral therapy; $\mathrm{AZT}=$ zidovudine; $\mathrm{NVP}=$ nevirapine; $3 \mathrm{TC}=$ lamivudine; $\mathrm{VL}=$ viral load; $\mathrm{PCR}=$ polymerase chain reaction; $\mathrm{cps} / \mathrm{mL}=$ copies per $\mathrm{mL}$ of blood.

infections so as to reduce early childhood morbidity and mortality. Current guidelines include routine HIV polymerase chain reaction (PCR) testing of all HIV-exposed neonates at birth, followed by a repeat HIV PCR at 10 weeks of age and 6 weeks after cessation of breastfeeding for those infants who previously tested negative. Infants who test positive should be fast-tracked within the treatment programme so that ART can be initiated within 7 days of diagnosis, with confirmatory HIV PCR testing performed on the same day. ${ }^{[7]}$

Despite having a robust evidence-based PMTCT policy framework, SA's progress towards eliminating mother-to-child transmission of HIV (eMTCT) is considerably challenged because of very high maternal HIV prevalence, ${ }^{[11]}$ including high rates of seroconversion during pregnancy and breastfeeding. ${ }^{[12,13]}$ Even with a low early MTCT transmission rate of $\sim 1.5 \%$ among HIV-infected mothers, ${ }^{[4]}$ recent laboratory data suggest that the national intrauterine infection rate is already five times higher than the WHO eMTCT target of $<50$ HIV-infected infants per 100000 live births. ${ }^{[1,15]}$ As intrapartum and postnatal transmission probably account for at least half of all MTCT, ${ }^{[16,17]}$ it is clear that SA has a considerable way to go before achieving eMTCT. Towards realising this end, the country must not only tackle the high HIV incidence among pregnant and breastfeeding women, particularly adolescent and young mothers (15 - 24 years), ${ }^{[18]}$ but also identify potential gaps in the PMTCT cascade and develop targeted interventions to reduce new paediatric HIV infections. Gaps identified in the PMTCT cascade that facilitate MTCT include late HIV diagnosis in pregnant women and subsequent lack of or delay in ART initiation during pregnancy. ${ }^{[13,19,20]}$ These factors preclude the sufficient time on ART required to suppress maternal viraemia to levels protective against MTCT risk during pregnancy, at delivery, and during the postpartum period while breastfeeding. Additional potential PMTCT gaps include lack of virological suppression among women already on ART, inadequate prophylaxis for exposed infants, and discontinuation of care by mother-infant pairs (MIPs) postpartum. ${ }^{[20]}$ Among social factors, youth pregnancy has been associated with an increased risk of MTCT. ${ }^{[18]}$

Mobile health (mHealth) technologies provide an opportunity for rapid identification of PMTCT gaps for intervention and subsequent improvement in service delivery. ${ }^{[2]}$ For infants who are already infected, improved case-finding can lead to accelerated entry into and retention in care. ${ }^{[22,23]}$ It is in response to the latter that automated HIV PCR Results for Action (RfA) reports from the National Health Laboratory Service (NHLS) Corporate Data Warehouse (CDW) have been routinely generated since June 2015. The HIV PCR RfA reports contain all laboratory-authorised HIV PCR results (including specimen reference numbers) for a particular region (e.g. health facility or district) and include patient demographic details (including name, surname, date of birth and age) and geographical location (health facility and patient contact details, including address and telephone number). These password-protected reports are routinely emailed on a weekly basis to PMTCT co-ordinators in the provincial and district health departments for active tracing and linkage to care of all HIV PCR-positive infants.

\section{Objectives}

To report findings from an operational study that aimed to describe, in near real time, potential reasons for MTCT among HIV-infected infants aged $<18$ months in three districts of KwaZulu-Natal (KZN) Province, SA.

\section{Methods}

\section{Study population}

The study was conducted among HIV-infected MIPs identified in three districts in KZN, namely eThekwini, uMgungundlovu and uMkhanyakude. In 2013, these three districts were among six districts with the highest antenatal burden of HIV in SA, each having a prevalence of $>40 \%{ }^{[11]}$ eThekwini and uMgungundlovu are large 


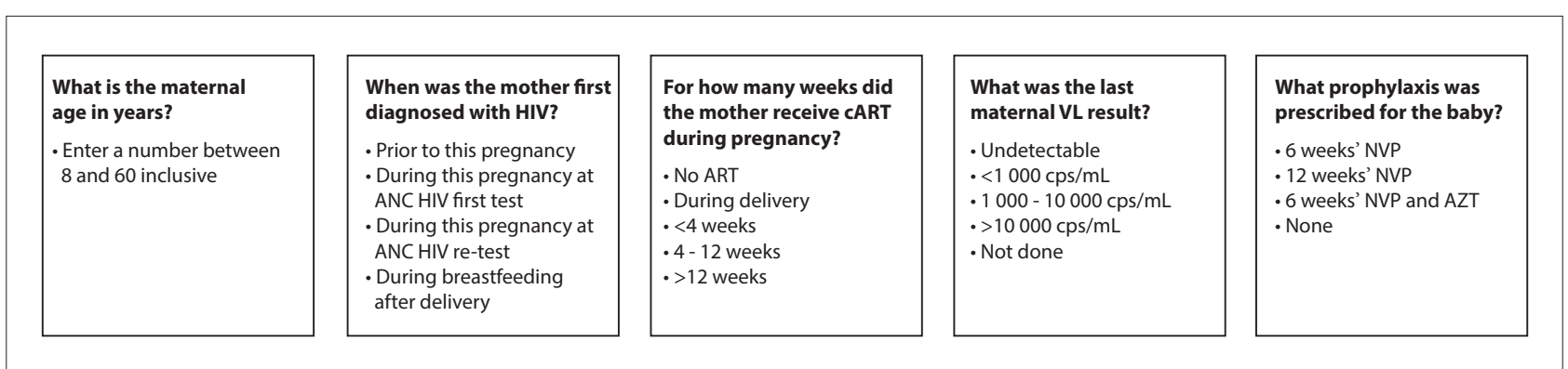

Fig. 1. The mobile phone-based questionnaire used to collect study data. $(A N C=$ antenatal care; $(c) A R T=($ combination $)$ antiretroviral therapy; VL $=v i r a l$ load; $c p s / m L=$ copies per $m L$ blood; $N V P=$ nevirapine; $A Z T=$ zidovudine. $)$

metropolitan districts, while uMkhanyakude is rural and one of the poorest districts in the country. ${ }^{[24]}$

\section{Study design and data collection}

This was an operational study in which a retrospective review of medical records of MIPs was conducted over a 5-month period from 1 May to 30 September 2016. For the purposes of this study, HIV PCR RfA reports from the NHLS CDW were emailed daily to the PMTCT co-ordinators and used to identify all HIV PCR-positive infants for linkage to care and inclusion in the study. MIP PMTCT data recorded in facility-based patient medical records and the national HIV electronic register (Tier. net) were retrospectively reviewed to answer five questions considered to be potential gaps for MTCT (Fig. 1).

The five questions focused on current maternal age, timing of maternal diagnosis in relation to the pregnancy, maternal ART treatment history, most recent maternal VL and the infant prophylaxis prescribed. Rapid Pro, an open-source communication platform that allows for collection of data via the mobile phone SMS (text message) service was used. The HIV PCR specimen reference number from the HIV PCR RfA report was used to identify linked MIP data. Once the PMTCT co-ordinators had been notified of an HIV PCR-positive infant in their district, they contacted the facility at which the infant's blood specimen had been collected and requested facility staff to provide answers to the questionnaire, or travelled to the facility and reviewed the records themselves. The district PMTCT co-ordinators then SMSed the HIV PCR specimen reference number to a short code number that triggered Rapid Pro to initiate the mobile phone-based questionnaire. Data for each HIV PCR-positive MIP were stored in Rapid Pro for near-real-time analysis. We aimed for a turnaround time of 3 working days from the time of notification of positive HIV PCRs by RfA reports to completing the questionnaire in Rapid Pro. Only the first HIV PCR-positive test per infant during the study period was included. Repeat confirmatory HIV PCR tests, indeterminate specimens (those that were neither positive nor negative) and tests registered outside the study period were excluded from enrolment.

\section{Statistical analysis}

Rapid Pro data were exported into Excel 2010 (Microsoft, USA), merged with HIV PCR RfA report data, and imported into Stata 14 (StataCorp, USA) for analysis. Descriptive statistics were conducted to describe the cohort. Continuous variables were described using medians and interquartile ranges (IQRs), while categorical data were described using proportions and compared using Pearson's $\chi^{2}$ test, or Fisher's exact test for sparse data.

\section{Ethics clearance}

The study was approved by the Human Research Ethics Committee of the University

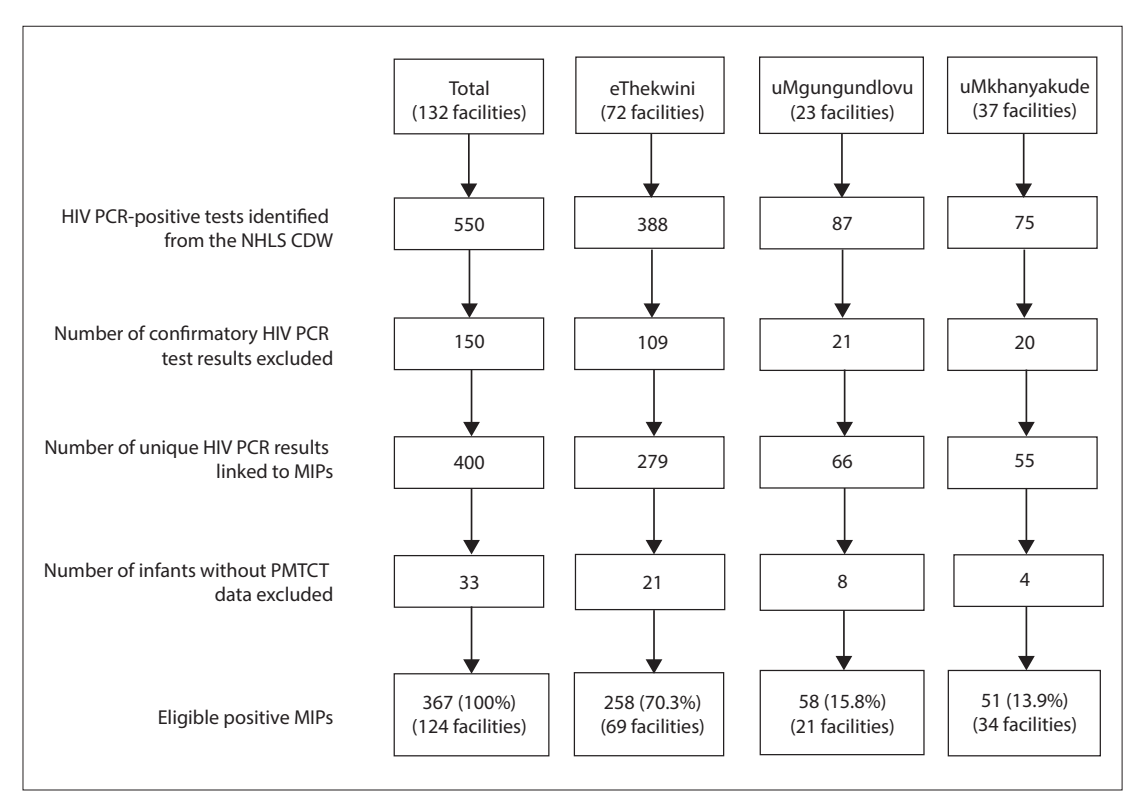

Fig. 2. Description of how the study cohort was obtained from the original dataset. $(P C R=$ polymerase chain reaction; NHLS CDW = National Health Laboratory Service Corporate Data Warehouse; $P M T C T=$ prevention of mother-to-child transmission of HIV; MIPs = mother-infant pairs.) of the Witwatersrand (ref. no. M160216) and the Health Research Committee of the KZN Department of Health (ref. no. HRKM100/16). Patient consent was not required, as the study was an audit of routine clinical care within a national healthcare programme. All information was de-identified prior to data analysis.

\section{Results}

\section{Mother-infant pairs}

Out of a total of 400 infants with positive HIV PCR test results, 367 (91.8\%) were included in the study as determined by available MIP PMTCT data (Fig. 2): 258 (70.3\%) from 69 facilities in eThekwini, 58 (15.8\%) from 21 facilities in uMgungundlovu and 51 (13.9\%) from 34 facilities in uMkhanyakude. Infant age at testing varied, with 178 (48.5\%) results performed at $<3$ months of age, 105 (28.6\%) infants having been tested within the first 6 days of life. Data were received within a median of 12.5 days (IQR 6 - 23) from the time district PMTCT co-ordinators 
were notified of a positive HIV PCR result, with $89.9 \%$ collected via SMS using Rapid Pro and the remainder via email.

\section{Prevention of mother-to-child transmission gaps}

Table 2 describes maternal and infant characteristics of the study cohort. The median age for mothers whose infants tested HIV PCRpositive was 25 years (IQR 22 - 30), with only 48 (13.1\%) being teenage mothers (15 - 19 years). The sample size was too small to determine whether there were significant differences in PMTCT gaps between the 48 teenage mothers and 293 older mothers (20 - 34 years).

Of the 367 women, $220(60.0 \%)$ were first diagnosed prior to conception or at their first antenatal care (ANC) visit, $14(3.8 \%)$ at a later ANC visit, and 127 (34.6\%) at or after delivery; in 6 cases (1.6\%) no information was available. Overall, 218 women $(59.4 \%)$ received ART prior to delivery, with $137(37.3 \%)$ receiving $>12$ weeks of therapy. Of 134 women (36.5\%) who did not receive ART, 17 (12.7\%) were diagnosed prior to conception or at their first ANC visit, 22 $(16.4 \%)$ at delivery and $95(70.9 \%)$ during breastfeeding.

A total of 110 women in the study (30.0\%) had a documented VL. Of these, $45(40.9 \%)$ were virologically suppressed as defined by a VL $<1000$ copies per mL. Of the 234 women known to be HIV-positive during ANC, 98 (41.9\%) had a documented VL and 41 (41.8\%) were virologically suppressed, the latter group accounting for $11.2 \%$ of all transmissions. Of 137 women who received $>12$ weeks of ART, 75 (54.7\%) had documented evidence of VL monitoring.

Of the $284(77.4 \%)$ infants with documented evidence that prophylaxis had been prescribed, 177 (62.3\%) and 107 (37.7\%) received low- and high-risk prophylaxis (as defined in Table 1), respectively. The majority of infants for whom no prophylaxis was prescribed were born to women diagnosed during breastfeeding. Among 30 infants (8.2\%) born to women diagnosed during delivery, and therefore known to be at increased risk for MTCT, 20 $(66.7 \%)$ received high-risk prophylaxis, $8(26.7 \%)$ received low-risk prophylaxis and $2(6.7 \%)$ received none.

We did not observe any statistically significant differences in responses to the PMTCT gap questions between the three districts, owing to small sample sizes in uMgungundlovu and uMkhanyakude; facility-level data were too sparse to demonstrate any differences either. The median number of PCR-positive tests per facility was 2 (IQR 1 - 3) for eThekwini, 1 (IQR 1 - 3) for uMgungundlovu and 1 (IQR 1 - 2) for uMkhanyakude. An analysis involving outlier facilities (facilities with PCR-positive tests greater than their district's 75th percentile number) showed no statistical differences in either

Table 2. Description of maternal and infant characteristics of the study cohort

\begin{tabular}{|c|c|c|c|c|}
\hline \multirow[b]{2}{*}{ Characteristic } & \multicolumn{3}{|c|}{ Maternal age (years) } & \multirow[b]{2}{*}{$p$-value } \\
\hline & Total $(N=367)$ & $15-19(n=48)$ & $20-34(n=293)$ & \\
\hline Age of infant at first HIV PCR-positive test (months) & & & & 0.11 \\
\hline Median (IQR) & $3.3(0.1-9.9)$ & $4.3(0.8-10.5)$ & $2.9(0.1-9.6)$ & \\
\hline$<3, n(\%)$ & $178(48.5)$ & $18(37.5)$ & $148(50.5)$ & \\
\hline $3-<6, n(\%)$ & $55(15.0)$ & $9(18.8)$ & $43(14.7)$ & \\
\hline $6-<12, n(\%)$ & $68(18.5)$ & $11(22.9)$ & $48(16.4)$ & \\
\hline $12-<18, n(\%)$ & $66(18.0)$ & $10(20.8)$ & $54(18.4)$ & \\
\hline Timing of maternal HIV diagnosis, $n(\%)$ & & & & 0.05 \\
\hline Prior to current pregnancy & $87(23.7)$ & $3(6.3)$ & $69(23.6)$ & \\
\hline During current pregnancy at ANC HIV first test & $133(36.2)$ & $23(47.9)$ & $106(36.2)$ & \\
\hline During current pregnancy at ANC HIV re-test & $14(3.8)$ & $1(2.1)$ & $13(4.4)$ & \\
\hline During current pregnancy at delivery & $30(8.2)$ & $3(6.3)$ & $26(8.9)$ & \\
\hline During breastfeeding & $97(26.4)$ & $18(37.5)$ & $74(25.3)$ & \\
\hline Unknown & $6(1.6)$ & 0 & $5(1.7)$ & \\
\hline Maternal treatment history, $n(\%)$ & & & & 0.29 \\
\hline No ART & $134(36.5)$ & $22(45.8)$ & $103(35.2)$ & \\
\hline ART during delivery & $7(1.9)$ & 0 & $6(2.1)$ & \\
\hline ART $<4$ weeks & $24(6.5)$ & $5(10.4)$ & $18(6.1)$ & \\
\hline ART 4 - 12 weeks & $57(15.5)$ & $8(16.7)$ & $45(15.4)$ & \\
\hline ART $>12$ weeks & $137(37.3)$ & $13(27.1)$ & $114(38.9)$ & \\
\hline Unknown & $8(2.2)$ & 0 & $7(2.4)$ & \\
\hline Last recorded maternal VL result (cps/mL), $n(\%)$ & & & & 0.24 \\
\hline Undetectable & $21(5.7)$ & 0 & $19(6.5)$ & \\
\hline$<1000$ & $24(6.5)$ & $2(4.2)$ & $21(7.2)$ & \\
\hline $1000-10000$ & $21(5.7)$ & $3(6.3)$ & $16(5.5)$ & \\
\hline$>10000$ & $44(12.0)$ & $6(12.5)$ & $33(11.3)$ & \\
\hline Did not have a documented VL & $257(70.0)$ & $37(77.1)$ & $204(69.6)$ & \\
\hline Infant prophylaxis, $n(\%)$ & & & & 0.53 \\
\hline 6 weeks' NVP & $177(48.2)$ & $21(43.8)$ & $143(48.8)$ & \\
\hline 12 weeks' NVP & $31(8.5)$ & $3(6.3)$ & $24(8.2)$ & \\
\hline 6 weeks' NVP + AZT & $76(20.7)$ & $11(22.9)$ & $60(20.5)$ & \\
\hline None & $72(19.6)$ & $13(27.1)$ & $57(19.5)$ & \\
\hline Unknown & $11(3.0)$ & 0 & $9(3.1)$ & \\
\hline
\end{tabular}


the proportions of outlier facilities $(p=0.57)$ or the median number of PCR-positive tests recorded (16 (6.2\%) for outlier facilities in eThekwini, $3(5.2 \%)$ in uMgungundlovu and $5(9.8 \%)$ in uMkhanyakude) $(p=0.07)$.

\section{Prevention of mother-to-child transmission cascade}

Fig. 3 illustrates the cascade of PMTCT services, demonstrating that $36.3 \%$ of HIV-positive infants were born to mothers diagnosed late, either at delivery or during breastfeeding, with an additional $26.6 \%$ of mothers receiving $<12$ weeks of ART prior to delivery. A further 1.5\% of these infants were not prescribed any antiretroviral prophylaxis. In total, 131 HIV transmissions (35.6\%) occurred despite both maternal and infant PMTCT care having been received.

\section{Discussion}

The findings of this study reveal critical gaps in the PMTCT cascade that require urgent intervention as SA works towards eMTCT. Whereas it is well documented that timing of maternal HIV diagnosis and subsequent access to ART are strong predictors of HIV transmission, ${ }^{[19,20,25-27]}$ our findings suggest that the majority of MTCT occurred among women who accessed HIV testing services at their first ANC visit, with a quarter already having been diagnosed prior to conception. At the opposite end of the spectrum, a third of infections occurred among women diagnosed at delivery or during breastfeeding. However, it could not be ascertained what proportion of these were due to incident HIV infections or failure to access timeous ANC, owing to data being unavailable in this study. Regarding treatment monitoring, only $30.0 \%$ of mothers had at least one VL result. Even among mothers who received $>12$ weeks of ART, only $54.7 \%$ had evidence of VL monitoring, with less than half of those with VL test results available being virologically suppressed. In total, $12.3 \%$ of transmitting mothers had documented evidence of VL suppression. As maternal VL is associated with risk of MTCT, ${ }^{[9]}$ and is used to inform the infant PMTCT regimen ${ }^{[7]}$ poor maternal treatment monitoring during the antenatal period jeopardises the success of the PMTCT programme.

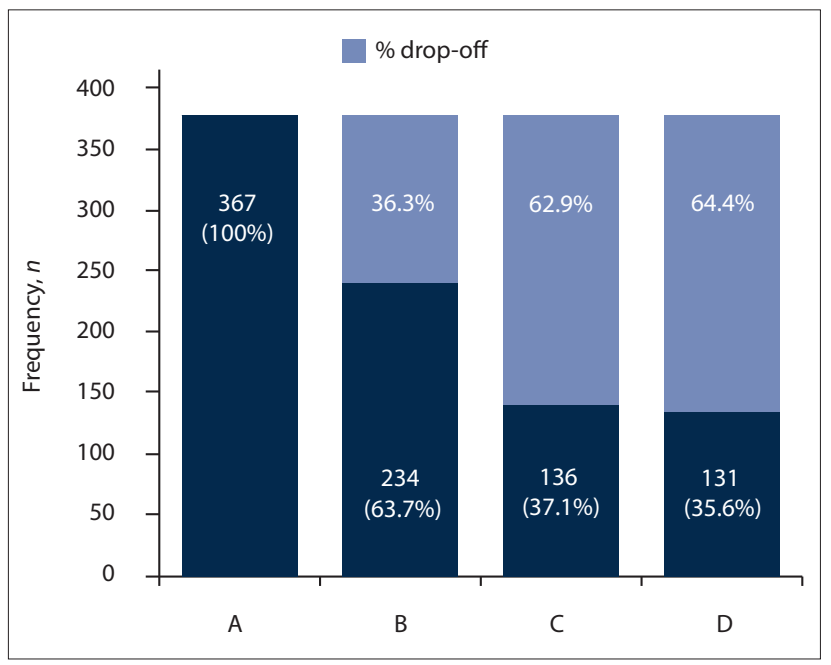

Fig. 3. Cascade of PMTCT services in the study cohort. This figure tracks the flow of HIV-positive MIPs along the continuum of PMTCT care in the three districts. $(A=$ total MIPs with positive infant HIV PCR; $B=$ total $M I P s$ where maternal HIV infection was diagnosed prior to pregnancy or during ANC (first HIV test and re-test); $C=$ total MIPs where maternal $A R T$ received for $>12$ weeks; $D=$ total infant prophylaxis where maternal $A R T$ received for $>12$ weeks; PMTCT $=$ prevention of mother-to-child transmission of HIV; MIPS = mother-infant pairs; $P C R=$ polymerase chain reaction; $A N C=$ antenatal care; $A R T=$ antiretroviral therapy.)
These findings are indicative of severe shortcomings in health services relating to the prevention and planning of pregnancies, management of HIV in women of reproductive age, and provision of optimal PMTCT care to MIPs. As VL monitoring during pregnancy has only recently been included in national guidelines, the lack of documented virological testing may represent slow uptake of current recommendations, which were previously focused on monitoring CD4+ counts. It may also reflect health system challenges, including poor record-keeping and result retrieval practices. However, the fact that infants born to newly diagnosed mothers were prescribed standard prophylaxis suggests that guidelines are not being adhered to, possibly owing to complexity of recommendations and poor understanding of risk stratification. It is anticipated that the availability of point-of-care VL testing during ANC and postnatal care may assist in the future by facilitating ready access to maternal VL testing and rapid turnaround time of results.

Although we aimed to receive PMTCT gap data within 3 working days, data were received only after a median of 12.5 days. Delays were attributed to technological failures (e.g. poor network coverage and delayed transfer of data into Rapid Pro) and poor recordkeeping at facility level. These results demonstrate that true realtime programme monitoring will require mHealth tools that are not dependent on continuous network availability and manual data entry. In addition, programme data will need to be centralised, with automated merging, analysis and reporting that feeds back to users timeously. Although near-real-time monitoring was not achieved, thereby potentially delaying efforts to identify MTCT high-burden areas and implement rapid targeted interventions, no differences in PMTCT gaps were observed between the three districts, or at facility level within each district. These results suggest that widespread enhancement of PMTCT services will be required if eMTCT is to be achieved. However, district sample volumes did not allow for sufficient power to determine whether differences were statistically significant. In addition, this study only included three districts in SA, so findings may not be representative of the country as a whole.

\section{Study limitations}

We acknowledge a number of limitations in this study. Whereas $\sim 50 \%$ of infants were first diagnosed at $<3$ months of age, with $28.6 \%$ diagnosed at or soon after birth, our results may not represent the true age distribution of HIV-infected infants, as we only included the first HIV PCR-positive test performed during the study period. We were therefore unable to ascertain whether infections were intrauterine, intrapartum or postpartum. Study findings were restricted to HIV-infected infants only, thereby precluding an HIVexposed uninfected control group for comparison. While younger maternal age has previously been associated with an increased risk of MTCT, ${ }^{[18,20,28]}$ we did not find significant differences in responses between teenage and older mothers on account of the small size of the study population and the lack of a control group. Furthermore, the number and depth of the questions asked in the study were limited by the data collection software used, resulting in too few data to explain why $36.2 \%$ of infants became infected despite being born to mothers who received $>12$ weeks of ART. For instance, we did not have data on maternal ART adherence, gestational age at first ANC visit, when maternal VL testing was performed in relation to delivery, or drug resistance profiles. We also did not have data on infant feeding or frequency of HIV testing during pregnancy to determine whether infections diagnosed late in pregnancy or during breastfeeding were incident infections or due to failure to access timeous ANC. Lastly, as this was a retrospective study, it was not possible to distinguish between what was documented and what actually occurred. Among 
women without evidence of VL monitoring, we therefore could not determine whether VLs were not done or simply not recorded at facility level or captured on Tier.net.

\section{Conclusions}

Two-thirds of the mothers who transmitted HIV to their infants did so despite receiving PMTCT services. The PMCT gaps outlined in this article highlight the need to improve quality of services during ANC as well as to prevent maternal infections postpartum. The focus of maternal PMTCT care needs to shift towards the prevention and planning of pregnancies as well as better management of HIV in women of reproductive age, including VL monitoring and intensified treatment adherence support. We intend using improved mHealth technologies to streamline data collection and reporting towards eMTCT.

Acknowledgements. The authors acknowledge the following PMTCT coordinators from the three study districts for assistance with data collection: Xolani Ndlovu, Dudu Khaba, Zinhle Ndlovu, Zamakhosi Mkhumane, Celiwe Mthembu and Dorcas Tembe. We are also grateful to Sue Candy and the NHLS CDW team for providing laboratory data.

Author contributions. GGS and SB framed and developed the design of the study as well as parameters for analysis. GGS, FM, OBM and BAS acquired and managed data collection from the field. FM, GGS, SB and TK analysed and interpreted the data. FM, AHM and GGS wrote the article. GGS, AHM, $\mathrm{SB}, \mathrm{OBM}, \mathrm{TK}$ and $\mathrm{KN}$ reviewed and approved the final version of the article. Funding. The authors acknowledge financial support for the study from UNICEF South Africa.

Conflicts of interest. None.

1. Barron P, Pillay Y, Doherty T, et al. Eliminating mother-to-child HIV transmission in South Africa. Bull World Health Organ 2013;91(1):70-74. http://doi.org/10.2471/BLT.12.106807

2. Sherman GG, Lilian RR, Bhardwaj S, et al. Laboratory information system data demonstrate successful implementation of the prevention of mother-to-child transmission programme in South Africa. S Afr implementation of the prevention of mother-to-child transmiss

3. Bhardwaj S, Treger-Slavin L, Barron P, et al. Elimination of mother-to-child transmission of HIV in South Africa: Rapid scale-up using quality improvement. S Afr Med J 2014;104(3):239-243. http://doi. org/10.7196/SAMJ.7605

4. Massyn N, Peer N, Padarath A, et al. District Health Barometer 2014/15. Durban: Health Systems Trust, 2015. https://www.health-e.org.za/wp-content/uploads/2015/10/Complete_DHB_2014_15_linked.pdf (accessed 27 February 2018).

5. National Department of Health, South Africa. Clinical Guidelines: Prevention of Mother-to-Child Transmission. Pretoria: NDoH, 2010. https://www.fidssa.co.za/Content/Documents/PMTCT_ Guidelines.pdf (accessed 27 February 2018)

6. National Department of Health, South Africa. The South African Antiretroviral Treatment Guidelines 2013: PMTCT Guidelines: Revised March 2013. Pretoria: NDoH, 2013. https://www.westerncape.gov. za/assets/ndoh_pmtct_guidelines.pdf (accessed 24 May 2017).

7. National Department of Health, South Africa. National Consolidated Guidelines for the Prevention of Mother-to-Child Transmission of HIV (PMTCT) and the Management of HIV in Children, Adolescents and Adults. Pretoria: NDoH, 2015. http://www.sahivsoc.org/Files/ART\%20 Guidelines\%2015052015.pdf (accessed 23 May 2017).
8. World Health Organization. Use of Antiretroviral Drugs for Treating Pregnant Women and Preventing HIV Infection in Infants. Geneva: WHO, 2012. http://www.who.int/hiv/PMTCT_update.pdf (accessed 4 May 2017).

9. Thea DM, Steketee RW, Pliner V, et al. The effect of maternal viralload on the risk of perinatal transmission of HIV-1. AIDS 1997;11(4):437-444. https://doi.org/10.1097\%2F00002030-199704000-00006

10. Roberts T, Cohn J, Bonner K, et al. Scale-up of routine viral load testing in resource-poor settings: Current and future implementation challenges. Clin Infect Dis 2016;62(8):1043-1048. http://doi. org/10.1093/cid/ciw001

11. National Department of Health, South Africa. The 2013 National Antenatal Sentinel HIV Prevalence Survey South Africa. Pretoria: NDoH, 2015. https://www.health-e.org.za/wp-content/ uploads/2016/03/Dept-Health-HIV-High-Res-7102015.pdf (accessed 23 May 2017).

12. Johnson LF, Stinson K, Newell M-L, et al. The contribution of maternal HIV seroconversion during late pregnancy and breastfeeding to mother-to-child transmission of HIV. J Acquir Immune Defic Syndr 2012;59(4):417-425. http://doi.org/10.1097/QAI.0b013e3182432f27

13. Dinh T-H, Delaney KP, Goga A, et al. Impact of maternal HIV seroconversion during pregnancy on early mother to child transmission of HIV (MTCT) measured at $4-8$ weeks postpartum in South Africa 2011 - 2012: A national population-based evaluation. PloS One 2015;10(5):e0125525. https:// Africa 2011 - 2012: A national popula

14. Sherman GG. Testing at birth - update from South Africa. Presented at the 8th HIV Pediatric Workshop, Durban, $15-16$ July 2016. http://regist2.virology-education.com/2016/8Pediatrics/05_ Sherman.pdf (accessed 27 February 2018).

15. World Health Organization. Global Guidance on Criteria and Processes for Validation: Elimination of Mother-to-Child Transmission (EMTCT) of HIV and Syphilis. Geneva: WHO, 2014. http://apps.who. int/iris/bitstream/10665/112858/1/9789241505888_eng.pdf?ua =1 \&ua =1 (accessed 22 May 2017)

16. Lilian RR, Kalk E, Bhowan K, et al. Early diagnosis of in utero and intrapartum HIV infection in infants prior to 6 weeks of age. J Clin Microbiol 2012;50(7):2373-2377. https://doi.org/10.1128/JCM.00431-12

17. Goga AE, Jackson DJ, Lombard C, et al. Highest risk of mother-to-child transmission of HIV or death in the first 6 months postpartum: Results from 18 month follow-up of an HIV-exposed national cohort, South Africa. Presented at the 21st International AIDS Conference, Durban, 18 - 22 July 2016. http://programme.aids2016.org/Abstract/Abstract/6477 (accessed 20 April 2017).

18. Fatti G, Shaikh N, Eley B, et al. Adolescent and young pregnant women at increased risk of mother-tochild transmission of HIV and poorer maternal and infant health outcomes: A cohort study at public facilities in the Nelson Mandela Bay Metropolitan district, Eastern Cape, South Africa. S Afr Med J facilities in the Nelson Mandela Bay Metropolitan district,

19. Technau K-G, Kalk E, Coovadia A, et al. Timing of maternal HIV testing and uptake of prevention of mother-to-child transmission interventions among women and their infected infants in Johannesburg, South Africa. J Acquir Immune Defic Syndr 2014;65(5):e170-e178. http://doi.org/10.1097/ QAI.000000000000006

20. Kendall C, Dorward J, Mfeka G, et al. Reasons for failure of prevention of mother-to-child HIV transmission in a rural South African district hospital. S Afr J HIV Med 2015;16(1):1-3. http://doi. org/10.4102/sahhivmed.v16il.365

21. Free C, Phillips G, Watson L, et al. The effectiveness of mobile-health technologies to improve health care service delivery processes: A systematic review and meta-analysis. PLoS Med 2013;10(1):e1001363. http://doi.org/10.1371/journal.pmed.1001363

22. Ibeto M, Giddy J, Cox V. Closing the gaps: Steps towards elimination of mother-to-child transmission of HIV. S Afr J HIV Med 2014;15(3):107-109. http://doi.org/10.7196/sajhivmed.1047

23. Woldesenbet SA, Jackson D, Goga AE, et al. Missed opportunities for early infant HIV diagnosis: Results of a national study in South Africa. J Acquir Immune Defic Syndr 2015;68(3):e26-e32. http:// doi.org/10.1097/QAI.0000000000000460

24. Day C, Barron P, Monticelli F, Sello E. Socio-economic indicators: Indicator comparisons by district. District Health Barometer 2007. http://www.hst.org.za/publications/District\%20Health\%20 Barometers/DHB0708.pdf (accessed 27 February 2018).

25. Stinson K, Boulle A, Coetzee D, et al. Initiation of highly active antiretroviral therapy among pregnant women in Cape Town, South Africa. Trop Med Int Health 2010;15(7):825-832. http://doi.org/10.1111/ j.1365-3156.2010.02538.x

26. Hoffman R, Black V, Technau K, et al. Effects of highly active antiretroviral therapy duration and regimen on risk for mother-to-child transmission of HIV in Johannesburg, South Africa. J Acquir Immune Defic Syndr 2010;54(1):35-41. https://doi.org/10.1097/qai.0b013e3181cf9979

27. Myer L. Initiating antiretroviral therapy in pregnancy: The importance of timing. J Acquir Immune Defic Syndr 2011;58(2):125-126. http://doi.org/10.1097/QAI.0b013e31822ad573

28. Ramraj T, Olorunju S, Jackson D, et al. Mother-to-child transmission of HIV amongst adolescents enrolled in three nationally representative surveys, South Africa, 2010, 2011 - 2012 and 2012 - 2013. Presented at the 8th International AIDS Society Conference Vancouver, 19 - 22 July 2015. http./(www. ias2015.org/WebContent/File/IAS 2015 MED2.pdf (accessed 27 February 2018). 